\title{
Low-dose ultraviolet radiation selectively degrades chromophore-rich extracellular matrix components
}

\author{
Michael J Sherratt, |* Christopher P Bayley,2 Siobhan M Reilly,1,3 Neil K Gibbs, ${ }^{3}$ Christopher EM Griffiths ${ }^{3}$ \\ and Rachel EB Watson ${ }^{3}$ \\ Regenerative Biomedicine, University of Manchester, Manchester Academic Health Science Centre, Manchester, UK \\ 2 Faculty of Life Sciences, University of Manchester, Manchester Academic Health Science Centre, Manchester, UK \\ 3 Dermatological Sciences, University of Manchester, Manchester Academic Health Science Centre, Manchester, UK
}

*Correspondence to: Michael I Sherratt, Regenerative Biomedicine, 1.58I Stopford Building, University of Manchester, Oxford Road, Manchester MI 3 9PT, UK e-mail: michael.j.sherratt@manchester.ac.uk

\begin{abstract}
Photoageing of human skin due to chronic exposure to ultraviolet radiation (UVR) is characterized histologically by extensive remodelling of the dermal elastic fibre system. Whilst enzymatic pathways are thought to play a major role in mediating extracellular matrix (ECM) degeneration in UV-exposed skin, the substrate specificity of UVR-up-regulated and activated matrix metalloproteinases (MMPs) is low. It is unclear, therefore, how such cell-mediated mechanisms alone could be responsible for the reported selective degradation of elastic fibre components such as fibrillin-1 and fibulin- 5 during the early stages of photoageing. Here we use atomic force microscopy (AFM) and scanning transmission electron microscopy (STEM) to demonstrate that physiologically attainable doses $\left(20-100 \mathrm{~mJ} / \mathrm{cm}^{2}\right)$ of direct UV-B radiation can induce profound, dose-dependent, changes in the structure of, and mass distribution within, isolated fibrillin microfibrils. Furthermore, using reducing and native PAGE in combination with AFM, we show that, whilst exposure to low-dose UV-B radiation significantly alters the macromolecular and quaternary structures of both UV chromophore (Cys, His, Phe, Trp and Tyr)-rich fibrillin microfibrils (fibrillin-1, 21.0\%) and fibronectin dimers (fibronectin, 12.9\%), similar doses have no detectable effect on UV chromophore-poor type I collagen monomers (2.2\%). Analysis of the published primary amino acid sequences of 49 dermal ECM components demonstrates that most elastic fibre-associated proteins, but crucially neither elastin nor members of the collagen family, are rich in UV chromophores. We suggest, therefore, that the amino acid composition of elastic fibre-associated proteins [including the fibrillins, fibulins, latent TGF $\beta$ binding proteins (LTBPs) and the lysyl oxidase family of enzymes (LOK/LOXLs)] may predispose them to direct degradation by UVR. As a consequence, this selective acellular photochemical pathway may play an important role in initiating and/or exacerbating cell-mediated ECM remodelling in UVR-exposed skin.

Copyright $\odot 2010$ Pathological Society of Great Britain and Ireland. Published by John Wiley \& Sons, Ltd.
\end{abstract}

Keywords: photoageing; skin; ultraviolet radiation; UV chromophores; elastic fibres; fibronectin; type I collagen; atomic force microscopy; scanning transmission electron microscopy

Received 6 February 2010; Revised 9 April 20 I0; Accepted 29 April 2010

No conflicts of interest were declared.

\section{Introduction}

In human skin, chronic exposure to ultraviolet radiation (UVR), the main cause of photoageing [1], induces profound changes in the architecture of the elastic fibre system in the dermis [1-3]. Elastic fibres are extracellular matrix (ECM) assemblies that permit long-range deformability and passive recoil in vertebrate tissues [4,5]. These composite fibres are composed primarily of a highly extensible elastin core [6] and an outer mantle of fibrillin-containing microfibrils, which are thought to direct elastic fibre formation [7], reinforce mature elastic fibres [8,9], transmit forces [10], support cell attachment [11] and mediate tissue homeostasis via sequestration of transforming growth factor $\beta$ (TGF $\beta$ ) [12].
Severely photoaged skin is characterized histologically by the deposition of disorganized elastotic material in the reticular dermis $[1,3]$, which replaces the normal elastic fibre architecture and is associated with a reduction in tissue elasticity [13]. In contrast, during the early stages of photoageing the elastic fibre components fibrillin-1 [14] and fibulin-5 [15] are lost from the microfibrillar apparatus at the dermal-epidermal junction (DEJ). Attempts to determine the causative mechanisms underlying changes in the mechanical properties of skin during photoageing have focused, primarily, on the failure of cells to maintain homeostasis via the aberrant expression of ECM proteases, such as the matrix metalloproteinases (MMPs) and their inhibitors [tissue inhibitors of metalloproteinases (TIMPs)] [16,17]. The substrate specificity of these proteases and inhibitors is, 
however, low $[18,19]$. Fibrillin microfibrils and peptides, for example, are catabolized by MMPs-2, -3, $-9,-12$ and -13 [20] and by the serine protease neutrophil elastase [21]. Furthermore, exposure of skin to low-dose UVR is sufficient to induce the expression of not only these enzymes but also MMPs-1, -7 and -9 $[3,22]$. As, collectively, these UV-up-regulated MMPs are capable of degrading more than 20 ECM proteins, including the abundant skin components collagen types I, III, IV and VII, fibronectin and elastin in addition to fibrillin [18], we suggest that enzyme-mediated pathways, in isolation, are unlikely to be responsible for the differential degradation of the microfibrillar apparatus in early photoageing.

In this study we initially employed quantitative ultrastructural and biochemical approaches to determine whether exposure to low-dose UVR in an acellular environment is sufficient to selectively alter the structure of ECM assemblies. Subsequently bioinformatic approaches were used to predict the relative susceptibility of individual ECM components to direct UVRmediated degradation.

\section{Methods}

\section{Reagents, tissue samples and cultured cells}

All reagents were obtained from Sigma-Aldrich Co. Ltd (Poole, UK) or BDH Ltd (Poole, UK), unless otherwise specified. A $6 \mathrm{~mm}$ diameter punch-biopsy (photoprotected buttock skin of a 23 year-old healthy male volunteer) was collected as part of a study into skin ageing approved by Salford and Trafford Research Ethics Committee (Ref. No. 02503). COS-1 cells were purchased from the European Collection of Cell Cultures (ECACC: Salisbury, UK).

\section{Fibrillin microfibril isolation}

COS-1 cells were maintained in DMEM (supplemented with $10 \%$ fetal calf serum and $50 \mu \mathrm{g} / \mathrm{ml}$ penicillin-streptomycin) at $37^{\circ} \mathrm{C}$ and $5 \% \mathrm{CO}_{2}$ until 3 weeks post-confluent. Fibrillin microfibrils were isolated and purified from cultured cells and human skin by bacterial collagenase digestion and size exclusion chromatography [8]. Briefly, tissue was incubated in $0.5 \mathrm{mg} / \mathrm{ml}$ type IA bacterial collagenase supplemented with protease inhibitors and $10 \mathrm{mM} \mathrm{CaCl} 2$ in column buffer (CB; $400 \mathrm{mM} \mathrm{NaCl}, 50 \mathrm{mM}$ Tris- $\mathrm{HCl}$, $\mathrm{pH}$ 7.4) for $4 \mathrm{~h}$ at $20^{\circ} \mathrm{C}$ with gentle agitation. Cultured cells were incubated with the same buffer on a rocking table for $3 \mathrm{~h}$ at $20^{\circ} \mathrm{C}$ prior to harvesting with a cell scraper. Digested material was centrifuged at $5000 \times g$ for $5 \mathrm{~min}$ prior to size fractionation on a CB-equilibrated Sepharose CL-2B column. Subsequent UV-B radiation exposure experiments were conducted on two pooled $1 \mathrm{ml}$ fractions from the centre of the excluded volume $\left(V_{0}\right)$ peak.

\section{UV-B irradiation of isolated ECM proteins and macromolecular assemblies}

All biomolecular suspensions were irradiated at a distance of $16 \mathrm{~cm}$ from under the centre of a bank of two $20 \mathrm{~W}$ Phillips TL-12 tubes (270-400 nm; Eindhoven, The Netherlands). UV-B (280-315 nm) irradiance was monitored using a UVX radiometer and UVX-31 detector (UVR Products, Upland, CA, USA), calibrated for the source with a spectroradiometer and standards traceable to the National Physical Laboratory (Teddington, UK). Cell and tissue microfibril suspensions were irradiated in $10 \mathrm{~mm}$ diameter $\times 2 \mathrm{~mm}$ high polyethylene dishes for varying time intervals to deliver incident UV-B radiation doses up to $50 \mathrm{~mJ} / \mathrm{cm}^{2}$ (human skin microfibrils), $100 \mathrm{~mJ} / \mathrm{cm}^{2}$ (COS-1 microfibrils) or $500 \mathrm{~mJ} / \mathrm{cm}^{2}$ (acid-soluble type I collagen and bovine plasma fibronectin). Absorbed UV-B (280-315 nm) doses, as determined by spectrophotometry of microfibril suspensions and subsequent convolution with the spectral output of the TL-12 source, were estimated to be $3 \%$ of incident UV-B doses.

Irradiated type I collagen suspensions in $0.5 \mathrm{M}$ acetic acid $(1 \mathrm{mg} / \mathrm{ml})$ were incubated with pepsin $(1 \mathrm{mg} / \mathrm{ml})$ for $30 \mathrm{~min}$ at room temperature. Following pepsinization, collagen suspensions were neutralized with $1 \mathrm{M}$ Tris. Fibronectin suspensions $(1 \mathrm{mg} / \mathrm{ml})$ were irradiated in a neutral, high-salt buffer $(500 \mathrm{mM} \mathrm{NaCl}$, $50 \mathrm{mM}$ Tris, $\mathrm{pH}$ 7.5).

\section{Quantitative ultrastructural analysis}

Fibronectin and fibrillin microfibrils were adsorbed from suspension onto mica and poly-L-lysine-coated mica, respectively, prior to visualization by intermittent contact mode atomic force microscopy (AFM; Nanoscope IIIa Multimode AFM, Veeco Instruments Inc., Santa Barbara, CA, USA) [8]. UVR-induced changes in microfibril mass and periodicity were quantified by dark-field scanning transmission electron microscopy (STEM; Tecnai 12 TWIN electron microscope, FEI Europe B.V., Eindhoven, The Netherlands) of microfibril suspensions adsorbed onto C-coated $\mathrm{Cu}$ grids [23]. The instrument was calibrated using a tobacco mosaic virus mass standard [24]. Mean mass maps were calculated for microfibril repeats with an axial periodicity of $57.9 \mathrm{~nm}$. For each experimental group, microfibril periodicity $(n=200)$, flexion angle $(n=500)$ and length $(n=50)$, and fibronectin height and area $(n=300)$ were determined from AFM images [8,25] by ImageJ [26], WSxM scanning probe microscopy software [27] and by routines written in Microsoft Visual Basic 6.0 [8].

\section{Protein sequence analysis}

The ProtParam tool (Swiss Institute of Bioinformatics: http://expasy.org/) was used to calculate the UV chromophore content (Cys, His, Phe, Tyr and Trp) [28] in the primary amino acid sequences of 49 processed dermal ECM components. Protein accession numbers, 
gene names, chain start/end positions and UV chromophore contents are detailed for collagens, proteoglycans/glycoproteins and elastic fibre components (see Supporting information, Tables S1-S3).

\section{Gel electrophoresis}

The influence of UV-B radiation on the apparent molecular weight of type I collagen and fibronectin was assessed by reducing SDS-PAGE on 6\% separating gels. Native PAGE on $6 \%$ gels was employed to characterize the effects of UV-B radiation exposure on the formation of protein complexes by type I collagen and fibronectin. Following PAGE, proteins were visualized by Coomassie blue staining.

\section{Results}

UV-B radiation exposure induces dose-dependent, structural changes in isolated fibrillin microfibrils

To investigate the susceptibility of fibrillin to UVR in vitro, we exposed microfibrils isolated from postconfluent fibroblast (COS-1) cell cultures to incident doses of UV-B irradiation (290-315 nm), which were less than $\left(20 \mathrm{~mJ} / \mathrm{cm}^{2}\right)$ equal to $\left(50 \mathrm{~mJ} / \mathrm{cm}^{2}\right)$ or greater than $\left(100 \mathrm{~mJ} / \mathrm{cm}^{2}\right)$, the mean incident dose causing minimal erythema (MED) in white skin [3]. It should be noted that the UV-B doses absorbed by the microfibril suspension were calculated, after convolution of spectrophotometric results with the spectral output of the TL-12 source, to be only $3 \%$ of the incident UVB doses. Following UVR exposure, extracted microfibrils appeared as uniform, semi-rigid, beaded filaments with a periodicity of $56 \mathrm{~nm}$ when visualized by AFM (Figure 1A). Exposure to UV-B radiation, however, significantly increased both microfibril periodicity (Figure 1B, G) and flexibility (Figure 1C, F) and induced fragmentation (Figure 1D, E) in a dosedependent manner.

In order to localize these UV-B-induced changes in macromolecular structure, the mass distribution within irradiated microfibrils was determined by STEM. In agreement with the AFM observations, physiologically attainable doses of UV-B irradiation induced multiple morphological changes in fibrillin microfibrils visualized by STEM (Figure 2A, B; see also Supporting information, Figure $\mathrm{S} 1$ ). The mass per repeat (MPR) of UV-B-irradiated microfibrils was significantly reduced (Figure 2C, D), with a UV-B dose of $100 \mathrm{~mJ} / \mathrm{cm}^{2}$ inducing a mass loss equivalent to more than $23 \%$ (322 $\mathrm{kDa})$ of the pre-irradiation mass (1358 \pm SEM $18.3 \mathrm{kDa})$. Structural reorganizations and/or mass losses were localized within the microfibril repeat by calculating mass difference maps from mean mass maps (Figure 2E, F). Mass was lost primarily from the central bead region of microfibrils exposed to UV$\mathrm{B}$ doses of 20 and $50 \mathrm{~mJ} / \mathrm{cm}^{2}$. Exposure to a UVB dose of $100 \mathrm{~mJ} / \mathrm{cm}^{2}$, however, induced profound
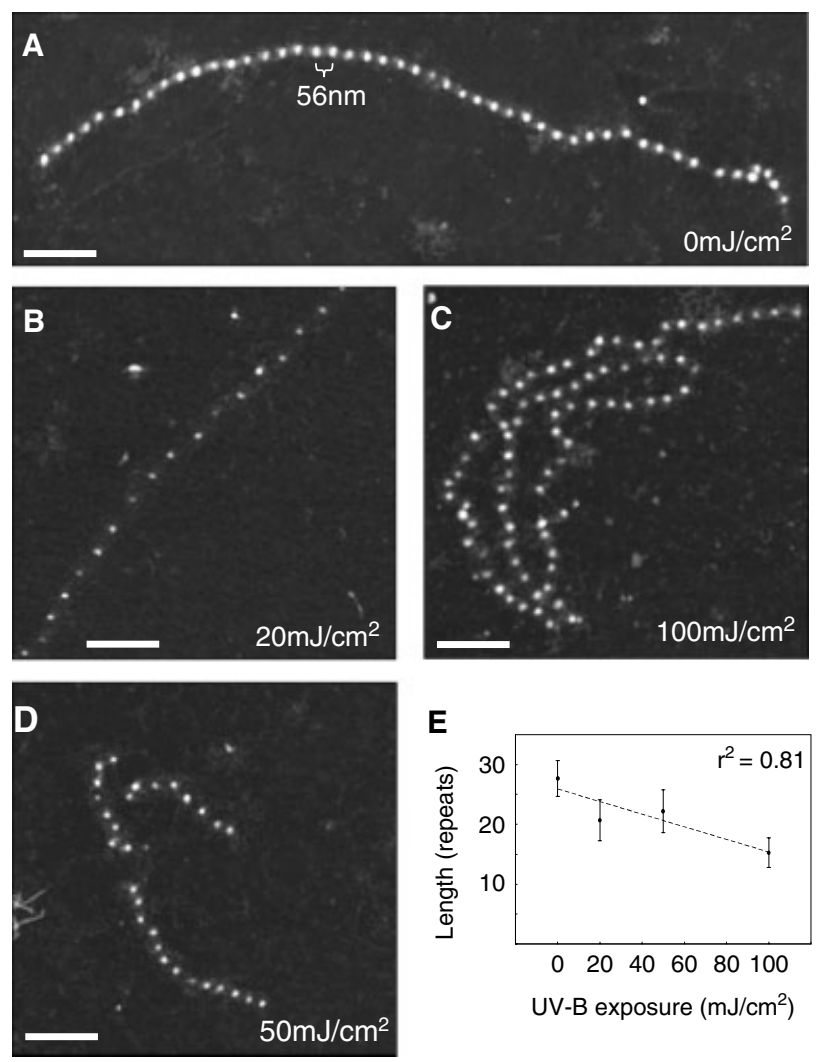

$\mathbf{F}$

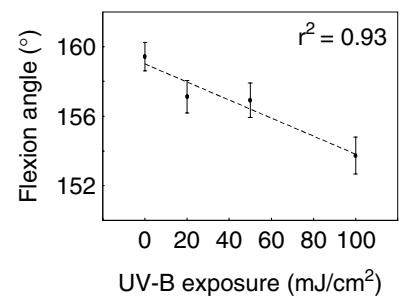

G

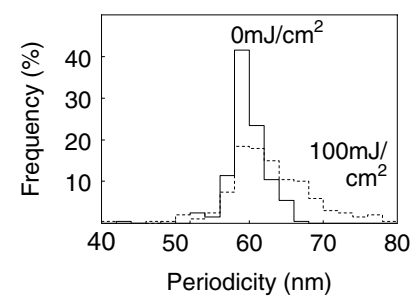

Figure 1. The influence of UV-B radiation on fibrillin microfibril morphology. (A-D) Intermittent contact mode AFM height images of non-exposed $(A)$ and UV-B radiation-exposed (B-D) COS-1 cell-derived fibrillin microfibrils. UV-B radiation induced profound changes in microfibril morphology, increasing periodicity (B) and flexibility (C) and inducing fragmentation (D). (E) Microfibril length was significantly reduced following exposure to increasing doses of $U V-B$ radiation $\left(p=0.0177,20 \mathrm{~mJ} / \mathrm{cm}^{2} ; p=0.0304,50 \mathrm{~mJ} / \mathrm{cm}^{2}\right.$; $p=0.0003,100 \mathrm{~mJ} / \mathrm{cm}^{2} ; n=50$, Mann-Whitney U-test). (F) UV$B$ radiation exposure significantly increased microfibril flexibility in a dose-dependent manner $\left(p=0.0114,20 \mathrm{~mJ} / \mathrm{cm}^{2} ; p=0.0286\right.$, $50 \mathrm{~mJ} / \mathrm{cm}^{2} ; p=0.0005,100 \mathrm{~mJ} / \mathrm{cm}^{2} ; n=500$, Mann-Whitney U-test). (G) Microfibril periodicity, as determined from STEM images, was significantly increased by exposure to $100 \mathrm{~mJ} / \mathrm{cm}^{2}$ UVR ( $p=6.5 \times 10^{-13}, n=200$, Mann-Whitney U-test). Dotted lines indicate linear regressions, error bars indicate SEM and scale bar $=200 \mathrm{~nm}$.

structural reorganizations within the whole microfibril repeat (Figure $2 \mathrm{~F}$ ).

\section{Both immature and mature microfibrils are degraded by UV-B radiation}

Whilst previous STEM investigations have established that, in adults, microfibril mass is relatively invariant between tissues and species [29], the mass of microfibrils derived from fetal tissues is positively correlated 

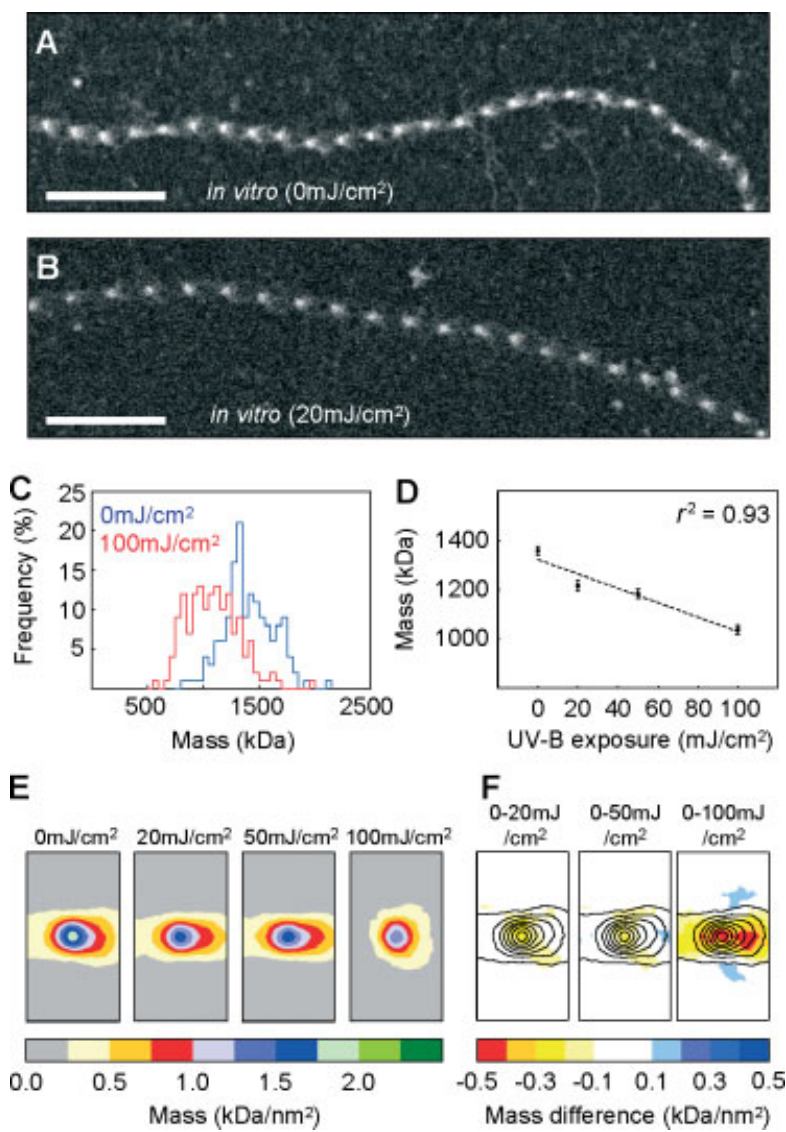

Figure 2. The influence of UV-B radiation exposure on the mass of COS-1 cell-derived microfibrils. $(A, B)$ Non-exposed (A) and UV-B radiation-exposed (B) COS-1 cell-derived fibrillin microfibrils visualized by STEM. As observed by AFM, UV-B radiation induced morphological alterations in both microfibril packing and periodicity. (C) Exposure of COS-1 cell-derived microfibrils to $100 \mathrm{~mJ} / \mathrm{cm}^{2}$ UV-B radiation significantly reduced MPR by $322 \mathrm{kDa}\left(p=3.9 \times 10^{-28} ; n=150\right.$, Student's $t$-test). (D) Exposure to lower doses of UV-B radiation significantly reduced microfibril MPR in a dose-dependent manner $(p=$ $6.6 \times 10^{-7}, 20 \mathrm{~mJ} / \mathrm{cm}^{2} ; p=4.9 \times 10^{-10}, 50 \mathrm{~mJ} / \mathrm{cm}^{2} ; n=150$, Student's t-test). (E) Mean mass maps derived from microfibrils exposed to increasing doses of UV-B radiation (axial periodicity $=57.9 \pm 2.5 \mathrm{~nm}$, box width/height $=103 / 58 \mathrm{~nm}, n=107$ $[20 \mathrm{~mJ}], 80[20 \mathrm{~mJ}], 115[50 \mathrm{~mJ}]$ and $66[100 \mathrm{~mJ}])$. Major UV$B$ radiation-induced structural rearrangements occurred between doses of $50 \mathrm{~mJ} / \mathrm{cm}^{2}$ and $100 \mathrm{~mJ} / \mathrm{cm}^{2}$. (F) Mass difference maps derived from mean mass maps [mass loss (yellow-red), mass gain (blue)]. The mass distribution within non-exposed microfibrils is indicated by contour lines. At lower UV-B radiation doses, mass was lost primarily from the centre of the microfibril bead. A $100 \mathrm{~mJ} / \mathrm{cm}^{2}$ dose, however, induced major structural rearrangements in axial packing in particular. The dotted line indicates linear regression; error bars indicate SEM; and scale bars $=200 \mathrm{~nm}$.

with developmental stage [30]. Here we demonstrate that the mean MPR of COS- 1 cell-derived microfibrils $(1358 \mathrm{kDa})$, is similar to previously reported MPRs of fetal tissue-derived microfibrils (Figure 3A). In order to exclude the possibility that only 'immature' microfibrils are susceptible to direct UVR-induced damage, we exposed microfibrils extracted from photoprotected adult human buttock skin to a UV-B radiation dose of $50 \mathrm{~mJ} / \mathrm{cm}^{2}$. Whilst exposure to UV-B radiation induced
A

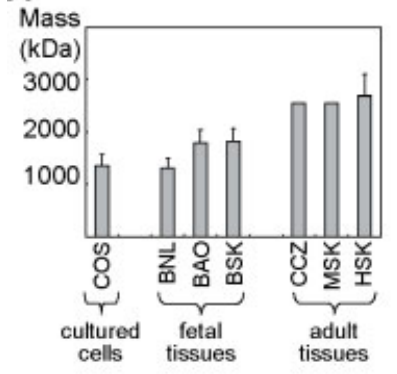

C

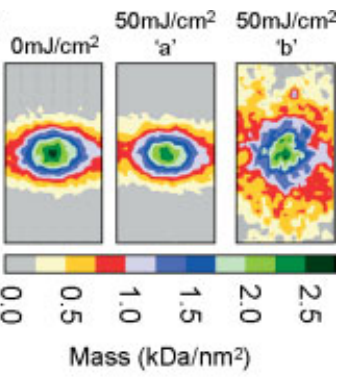

B

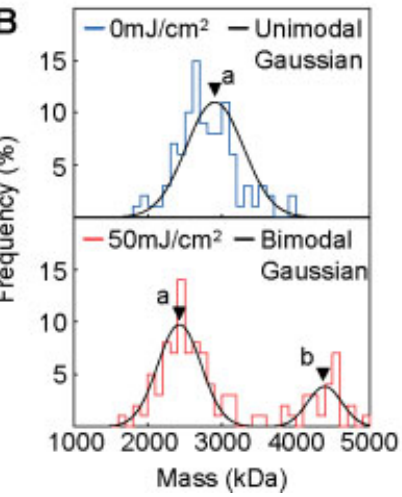

D $0-50 \mathrm{~mJ} / 0-50 \mathrm{~mJ} /$

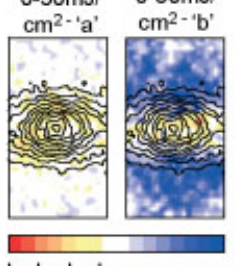

헝ㅇㅇㅇㅇㅇㅇ

Mass difference

$\left(\mathrm{kDa} / \mathrm{nm}^{2}\right)$

Figure 3. Tissue-derived microfibrils are degraded by direct exposure to UVR. (A) STEM-derived mean MPR of fibrillin microfibrils isolated from cultured cells [COS-1 cells (COS) $1358 \mathrm{kDa}$, this study], fetal tissues [bovine nuchal ligament (BNL) $1320 \mathrm{kDa}$, aorta (BAO) $1792 \mathrm{kDa}$ and skin (BSK) $1814 \mathrm{kDa}$ [ [30] and adult tissues [canine ciliary zonule (CCZ) $2550 \mathrm{kDa}$, murine skin (MSK) $2547 \mathrm{kDa}$ [29] and human skin (HSK) $2697 \mathrm{kDa}$, this study]. (B) Prior to UV-B radiation exposure, mean tissue microfibril MPR fitted a unimodal Gaussian distribution, which peaked at $2695 \mathrm{kDa}$ (population a). Following UV-B radiation exposure, mean microfibril MPR fitted a bimodal Gaussian distribution which peaked at $2429 \mathrm{kDa}$ (population a) and $4396 \mathrm{kDa}$ (population b). (C) Mean mass maps derived from non-exposed microfibrils and microfibrils exposed to $50 \mathrm{~mJ} / \mathrm{cm}^{2} \mathrm{UV}-\mathrm{B}$ radiation [axial periodicity $=57.9 \mathrm{~nm} \pm 2.5 \mathrm{~nm}$; box width/height $=103 / 58 \mathrm{~nm} ; n=37$ $(0 \mathrm{~mJ}), 31(50 \mathrm{~mJ}$ a) and $7(50 \mathrm{~mJ}$ b)]. (D) Mass difference maps derived from mean mass maps [mass loss (yellow-red), mass gain (blue)]. The non-exposed mass distribution is indicated by contour lines. Exposure to $50 \mathrm{~mJ} / \mathrm{cm}^{2}$ UV-B radiation induced mass loss throughout population a microfibril repeats. In contrast, population $b$ repeats were characterized by both axial mass loss and the profuse lateral accretion of disorganized material. Dotted line indicates linear regression; error bars indicate SEM; and scale bars $=200 \mathrm{~nm}$.

significant mass loss from the majority of microfibril repeats, a subpopulation of repeats additionally accreted substantial amounts of disorganized, laterally associated, material (Figure 3B-D; see also Supporting information, Figure S1). Exposure to physiologically attainable UV-B doses, equivalent to or less than the MED of $30-50 \mathrm{~mJ} / \mathrm{cm}^{2}$ [3] is sufficient, therefore, to directly induce profound changes in the structure of both in vitro (immature) and in vivo (mature) synthesized microfibrils.

\section{Elastic fibre-associated proteins are rich in UV-labile amino acids}

In combination, the observations that: (a) the elastic fibre system is selectively remodelled in photoaged 
skin $[1,3,14,15]$; and (b) UVR exposure profoundly affects the structure of isolated fibrillin microfibrils (this study) imply that elastic fibre components may be particularly photo-labile. UV chromophores are molecules, or regions of molecules, which absorb UV-B radiation. This absorbed energy may subsequently alter molecular structures, either directly, or indirectly via the generation of reactive oxygen species (ROS) [31]. As only a subset of the 20 amino acid residues found in human proteins (Trp, Tyr, Phe, His and Cys) act as UV-B chromophores [28], it follows that chemically distinct ECM molecules may exhibit differential susceptibilities to direct UVR.

We initially quantified the content of these five UV$\mathrm{B}$ chromophores within the published structures of two contiguous calcium-binding epidermal growth factorlike (cbEGF) domains [32] and a single TGF $\beta 1$-binding protein-like (TB) domain [33] found in fibrillin-1 (Figure 4A). Both domains, which dominate the structure of the fibrillins, are stabilized by multiple Cys-Cys (cystine) intra-domain disulphide bonds [29]. These bonds may be photodegraded directly, through absorption of UV-B radiation, or indirectly by electron capture from Trp or Tyr residues, which in turn can initiate an amino acid-degrading radical cascade [34]. As a consequence, primarily of the Cys content therein, over $20 \%$ of the amino acids within fibrillin-1 are UV chromophores (Figure 4B). Analysis of the published primary amino acid sequences of 49 key dermal ECM proteins indicates that a high UV chromophore content is a feature of not only the fibrillins (both-1 and -2) but also other major families of elastic fibre proteins, including fibulins, latent TGF $\beta$ binding proteins (LTBPs) and the lysyl oxidase family of enzymes (LOK/LOXLs) (Figure 4C). Collectively, therefore, elastic fibre-associated proteins (with the exception of elastin itself) are highly enriched in UV chromophores compared with ECM collagens and proteoglycans (Figure 4C).

The susceptibility of ECM components to direct UV-B radiation is positively correlated with their UV chromophore composition

Having already demonstrated that UV-B chromophorerich fibrillin microfibrils (fibrillin-1 UV chromophore content, $21.0 \%$ ) are susceptible to relatively low doses of UV-B radiation, we exposed suspensions of two abundant and functionally crucial dermal ECM components (type I collagen, UV chromophore content $2.2 \%$; fibronectin, UV chromophore content $12.9 \%$ ) to increasing doses of UV-B radiation. Non-irradiated type I collagen was visualized by reducing SDS-PAGE as distinct $\alpha 1(\mathrm{I})(130 \mathrm{kDa})$ and $\alpha 2(\mathrm{I})(116 \mathrm{kDa})$ procollagen bands (Figure 5Ai). Exposure to doses of UV-B radiation up to 10 times the MED $\left(500 \mathrm{~mJ} / \mathrm{cm}^{2}\right)$ [3] had no measurable effect on the electrophoretic mobilities or the relative intensities of UV chromophore-poor collagen I $\alpha$-chains (Figure 5A). Furthermore, exposure to UV-B radiation had no discernable effect on
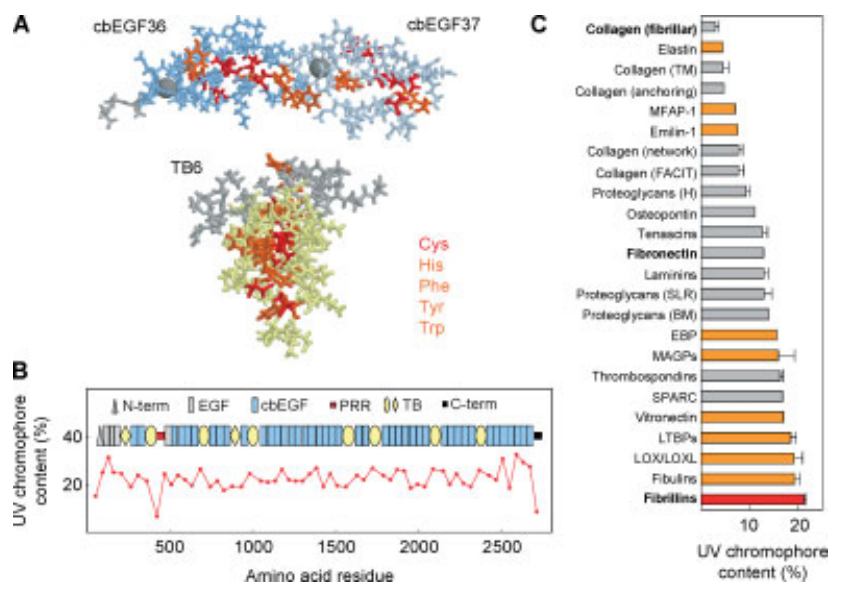

Figure 4. UV chromophore content and UVR susceptibility in ECM proteins. (A) Location of the UV chromophores Cys, His, Phe, Tyr and Trp within stick models of the major structural motifs of fibrillin-1 (grey spheres indicate bound $\mathrm{Ca}^{2+}$ ). Of the 79 residues in this cbEGF domain pair [32], 18 (22\%) are UV chromophores (12 Cys, 3 Phe, 2 Tyr and $1 \mathrm{His}$ ). The TB domain of fibrillin-1 is also rich in UV chromophores (15/72 residues; 21\%; 8 Cys, 1 His, 2 Phe, 3 Tyr, 1 Trp) [33]. (B) Distribution of UV chromophores within the domain structure of fibrillin-1 [derived from FBN1 (Acc. No. P35555)]. (C) Relative UV chromophore content of 49 dermal ECM proteins grouped according to structural similarity. UV chromophore content varied from 2.9\% (SEM 0.7\%) for the fibrillar collagens (I, III and V) to $21.3 \%$ (SEM $0.4 \%$ ) for members of the fibrillin family $(-1$ to -3). Elastic fibre and microfibril proteins are highlighted in orange or red (fibrillins). The composition of these family groups and the accession numbers, gene names, chain positions and UV chromophore contents of their constituent proteins are detailed in the Supporting information (Tables S1-S3). Error bars indicate SEM.

the quaternary structure of the type I collagen triple helix (Figure 5B).

In contrast, UV-B radiation promoted the formation of high-molecular weight (MW) fibronectin aggregates $\left(\mathrm{Ag}\right.$ and $\left.\mathrm{Ag}^{\prime}\right)$ and reduced the intensity of lower MW bands (Fn and $\mathrm{Fn}^{\prime}$ ) in a dose-dependent manner in both reducing and native conditions (Figure 5C, D; see also Supporting information, Figures S2-S4). Molecular fragmentation, however, was not observed, even at relatively high doses of UV-B radiation (see Supporting information, Figure S2). Fibronectin dimers form highly ordered structures in solution, which may be visualized by AFM as three globular domains connected by two extended arms [35]. Prior to UV-B radiation exposure, bovine plasma fibronectin appeared in both dimeric and monomeric forms (Figure 6A, C). Exposure to UV-B irradiation disrupted the ordered morphology of fibronectin, inducing aggregation (Figure 6C) and significantly altering both molecular height (Figure 6D) and area (see Supporting information, Figure S5).

Therefore, whilst UV-B chromophore-poor type I collagen $(2.2 \%)$ is unaffected by UVR doses of up to 10 times the MED $\left(500 \mathrm{~mJ} / \mathrm{cm}^{2}\right)$, the macromolecular and quaternary structures of fibronectin (UV chromophores, $12.9 \%$ ) are profoundly altered by exposure to multiple-MED UV-B doses $\left(100-500 \mathrm{~mJ} / \mathrm{cm}^{2}\right)$. Crucially, fibrillin microfibrils, which have a higher 
A

UVR $\left(\mathrm{mJ} / \mathrm{cm}^{2}\right)$

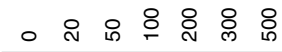

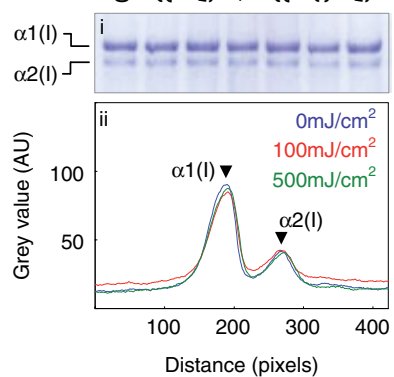

B

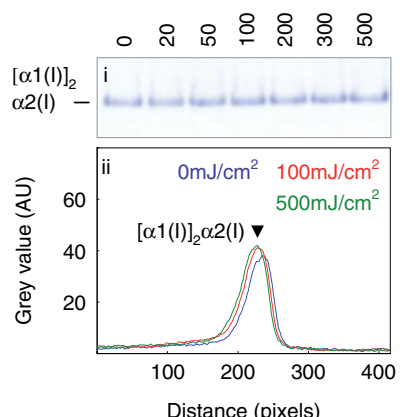

C

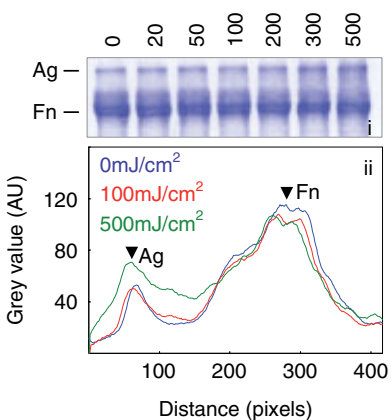

D

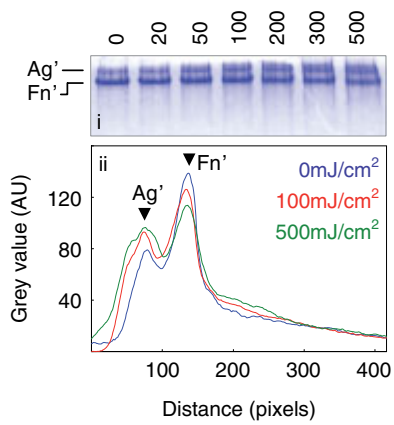

Figure 5. The influence of UVR exposure on the electrophoretic mobility and quaternary structure type I collagen monomers and fibronectin dimers. (A) Reducing SDS-PAGE of non-exposed and UV-B irradiated type I collagen. Electrophoresis in denaturing conditions disrupted the collagen triple helix $[\alpha 1(I)] 2 \alpha(I)]$ releasing the constituent $\alpha 1(I)$ and $\alpha 2(I)$ chains. UV-B radiation had no effect on the mobility or relative intensity of the $\alpha$ chain bands [i, Coomassie blue-stained polyacrylamide gel; ii, densitometric analysis of non-exposed $(0 \mathrm{~mJ})$ and UV-B-irradiated (100 and $500 \mathrm{~mJ}$ ) type I collagen]. (B) Native PAGE of non-exposed and UV-B-irradiated type I collagen. Electrophoresis by this method preserves intermolecular interactions and the mobility and relative intensity of the triple helical $[\alpha 1(I)] 2 \alpha(I)]$ band was unaffected by exposure to UV-B radiation. (C) Electrophoretic analysis by reducing SDS-PAGE of non-exposed and UV-B-irradiated bovine plasma fibronectin. ( $\mathrm{i}$ and ii) Coomassie blue-stained polyacrylamide gels and densitometric analyses of non-exposed $(0 \mathrm{~mJ})$ and UV-B irradiated (100 and $500 \mathrm{~mJ}$ ) fibronectin. UV-B irradiation reduced the intensity the 216-225 kDa monomeric fibronectin doublet (Fn) [49] and increased the intensity of a higher MW aggregate (Ag). (D) When visualized by native PAGE, UV-B irradiation altered the relative intensities of two bands designated $\mathrm{Fn}^{\prime}$ and $\mathrm{Ag}^{\prime}$.

UV chromophore content $(21.0 \%)$ than fibronectin, undergo extensive degradation at lower UV-B doses $\left(<50 \mathrm{~mJ} / \mathrm{cm}^{2}\right)$.

\section{Discussion}

The key elastic fibre component fibrillin is selectively lost during photoageing [14] and in this study we demonstrate that fibrillin microfibrils may be degraded in vitro by physiologically relevant doses of UVR. We suggest, therefore, that direct UVR exposure may provide an alternative in vivo catabolic pathway which, crucially, is more specific for Cys-rich elastic fibre components than enzyme-mediated proteolysis.
A

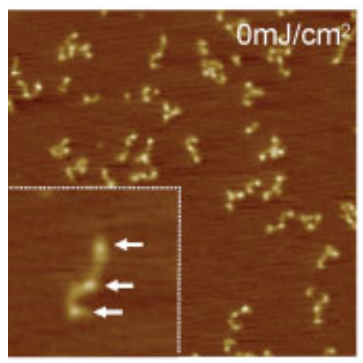

C

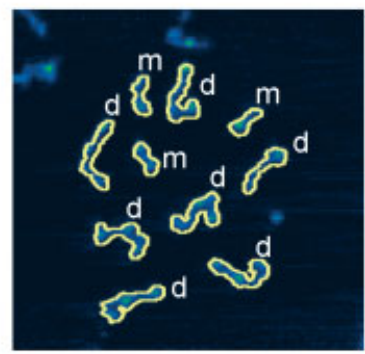

B
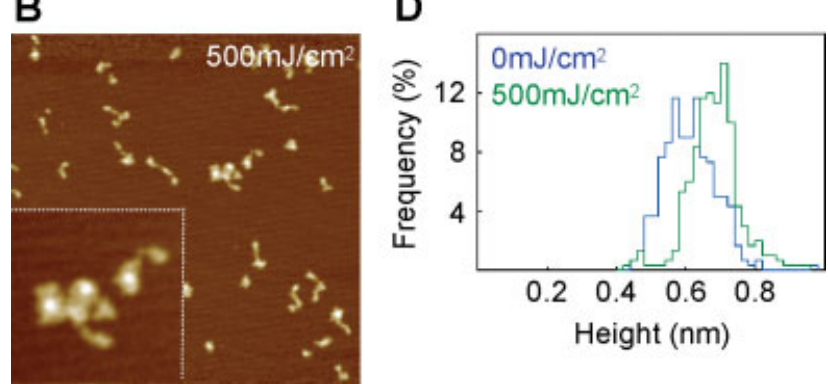

Figure 6. Exposure to UVR induces profound changes in the macromolecular structure of fibronectin. (A-C) AFM height images of non-exposed $\left(A, C ; 0 \mathrm{~mJ} / \mathrm{cm}^{2}\right)$ and $U V-B$ irradiated $\left(B ; 500 \mathrm{~mJ} / \mathrm{cm}^{2}\right)$ fibronectin suspensions $(1 \times 1 \mu \mathrm{m}, 3 \mathrm{~nm} \mathrm{z}$ scale; inset $200 \times 200 \mathrm{~nm}$ ). (C) Isodata thresholding and particle analysis applied to AFM height image data of non-exposed fibronectin adsorbed onto a mica surface $(500 \times 500 \mathrm{~nm}$, $3 \mathrm{~nm} z$ scale). Particles identified as dimers (d) occupied twice the surface area of monomers $(\mathrm{m})$. Abundant, tri-lobed (arrows), fibronectin dimers [35] were observed in the nonexposed population (A). Exposure to UV-B (B) disrupted this distinct morphology and promoted the formation of disordered aggregates. (D) Height frequency distributions of non-exposed $\left(0 \mathrm{~mJ} / \mathrm{cm}^{2}\right)$ and UV-B-irradiated $\left(500 \mathrm{~mJ} / \mathrm{cm}^{2}\right)$ fibronectin particles. Exposure to UV-B significantly increased the mean fibronectin particle height from $0.59 \pm 0.004 \mathrm{~nm}^{2}\left(0 \mathrm{~mJ} / \mathrm{cm}^{2}\right)$ to $0.67 \pm$ $0.004 \mathrm{~nm}\left(500 \mathrm{~mJ} / \mathrm{cm}^{2}\right)\left(p=5.4 \times 10^{-27} ; n=300\right.$, Student's $t$-test).

The unique tissue location, longevity and chemical composition of elastic fibre components may act synergistically to promote their degradation by direct UVR. Fibrillin-rich microfibrils, which form the cascade of oxytalan fibres in the papillary dermis [4], for example, will receive not only a higher total UVR dose [36] than fibrillar collagens and elastic fibres within the reticular dermis but also a relatively higher dose of less penetrating, but more biologically active, UVB [37]. Furthermore, whether located in oxytalan fibre cascades at the DEJ [14] or on the surface of elastic fibres [4], fibrillin microfibrils are more accessible to incident UVR than either elastin or type I collagen which form densely packed, large-diameter fibres and fibrils [4,38].

The temporal exposure of elastic fibre proteins may also exceed that of other tissue components. Whilst intracellular proteins have half-lives measured in hours [39], ECM assemblies have very low turnover rates in vivo [5]. In comparison with dermal collagens with an estimated half-life of 15 years [40], aspartic acid racemization and radio-labelling studies suggest that elastic fibre proteins may persist for the lifetime of 
an individual $[41,42]$. The sensitivity of microfibrils to low-dose UV-B irradiation, as demonstrated in this study, combined with their unique architecture and marked tissue longevity, provides ample opportunity for these structures to accumulate environmental damage as a consequence of chronic UVR exposure.

Finally, although specific morphological changes may be induced in microfibrils following exposure to $\mathrm{Ca}^{2+}$-chelating agents, which decrease periodically, or to proteases such as MMP-2 and -12, which cause fragmentation and increase periodicity respectively $[20,43]$, exposure to UVR appears to be unique in inducing manifold structural changes within the same molecular population. The variety of these morphological changes, when considered in conjunction with the generalized structural mass loss, implies that UV-B irradiation interacts stochastically with UV chromophores distributed throughout the fibrillin-1 molecule. We suggest, therefore, that the widespread distribution of cystine bonds and electron-donating Trp and Tyr residues (see Supporting information, Figure S6) within the primary structures of cbEGF-based microfibrillar proteins, such as the fibrillins, fibulins and LTBPs, may underlie the susceptibility of the microfibrillar apparatus to UVR exposure. In marked contrast, UV chromophorepoor type I collagen, which plays a key role in skin structure, is markedly insensitive to physiologically attainable UVR (Figure 5A, B). As early as 1965, Cooper and Davidson demonstrated that UVR (at wavelengths $<400 \mathrm{~nm}$ ) fragmented soluble collagen [44]. In more recent studies, UVR has been shown to influence collagenase-mediated collagen degradation [45], collagen fibrillogenesis [46] and the structure of the collagen helix [47]. In all of these studies, however, structural changes were induced only after exposure to radiation doses which were 200-5000 times greater than the highest dose used in this study. The structure of fibronectin (UV chromophore content 12.9\%), however, appears to be extremely sensitive to direct UVR exposure. As a consequence, UVR may profoundly affect cell-matrix interactions in vivo.

Regardless of the mechanisms underlying UVRinduced ECM damage, changes in fibrillin microfibril structure are likely to have profound biomechanical and biochemical consequences for tissue function. Within skin, reduced microfibrillar tensile strength, as a result of cleavage events, may impact on the ability of microfibrils to reinforce the elastic fibre and to transmit forces between the extracellular and cellular fractions of the tissue. Biochemically, structural alterations in both microfibril and fibronectin morphology, similar to those induced by UVR exposure, are sufficient to inhibit integrin-mediated cell attachment $[25,48]$. Alterations in surface charge or mass distribution have the potential to reduce the affinity of fibrillin for other ECM molecules, such as MAPG1, tropoelastin, decorin, fibulins and versican [4], and to increase the affinity of fibrillin for itself. It may be that this latter tendency for UVR to promote fibrillin fragmentation and self-association underlies not only the early loss of oxytalan fibres from the DEJ but also the later accumulation of dystrophic elastotic material in chronically photoaged skin [1]. Finally, disruption of the ability of fibrillin microfibrils to sequester TGF $\beta$ [12] is thought have profound consequences for tissue homeostasis. By interfering with both ECM homeostasis [3] and elastic fibre deposition [7], UVR-induced changes in fibrillin microfibril structure may, therefore, trigger uncontrolled elastic fibre remodelling.

In summary, since exposure to UVR increases the expression and activity of ECM-degrading MMPs [1], it has been assumed that matrix remodelling in photoaged skin is due primarily to enzymatic cleavage. Here we demonstrate that irradiation of isolated ECM molecules with physiologically attainable doses of UVR can selectively denature the molecular and macromolecular structures of UV chromophorerich species. Although it remains to be determined whether UVR-induced microfibrillar degradation is evident in vivo, we suggest that the location, architecture, low turnover and chemical composition of microfibrillar proteins all play a role in enhancing the susceptibility of these structures to direct UVR damage and that this direct damage may be an important mechanism in the process of skin photoageing.

\section{Acknowledgment}

We would like to thank Dr Helen Graham and Dr David Holmes for useful discussions of the data. We are also indebted to Mr Jean Bastrilles and Mrs June Bowden for their assistance with the collection of human skin samples. This work was supported by Research into Ageing as part of a Senior Fellowship awarded to MJS (Grant No. 266). SMR was supported by the Arthritis Research Campaign. Fibrillin microfibril purification was carried out on equipment purchased as part of a grant from the British Skin Foundation (Grant No. S204). CEMG is partly supported by the NIHR Manchester Biomedical Research Centre.

\section{Author contributions}

MJS, REBW and NKG conceived the experiments, which were subsequently carried out by SMR, CPB, MJS and NKG. MJS, NKG, SMR and CPB analysed the data and the paper was written by MJS, REBW, CEMG and NKG. All authors had final approval over both the submitted and published versions.

\section{References}

1. Yaar M, Gilchrest BA. Photoageing: mechanism, prevention and therapy. Br J Dermatol 2007; 157: 874-877.

2. Griffiths CEM, Russman AN, Majmudar G, et al. Restoration of collagen formation in photodamaged human skin by tretinoin (retinoic acid). N Engl J Med 1993; 329: 530-535. 
3. Fisher GJ, Datta SC, Talwar HS, et al. Molecular basis of suninduced premature skin ageing and retinoid antagonism. Nature 1996; 379: 335-339.

4. Kielty CM, Sherratt MJ, Shuttleworth CA. Elastic fibres. J Cell Sci 2002; 115: 2817-2828.

5. Sherratt MJ. Tissue elasticity and the ageing elastic fibre. Age 2009; 31: 305-325

6. Aaron BB, Gosline JM. Elastin as a random-network elastomer: a mechanical and optical analysis of single elastin fibers. Biopolymers 1981; 20: 1247-1260.

7. Carta L, Pereira L, Arteaga-Solis E, et al. Fibrillins 1 and 2 perform partially overlapping functions during aortic development. J Biol Chem 2006; 281: 8016-8023.

8. Sherratt MJ, Baldock C, Haston JL, et al. Fibrillin microfibrils are stiff reinforcing fibres in compliant tissues. J Mol Biol 2003; 332: 183-193.

9. Carton RW, Dainauskas J, Clark JW. Elastic properties of single elastic fibers. J Appl Physiol 1962; 17: 547-551.

10. Ashworth JL, Kielty CM, McLeod D. Fibrillin and the eye. $\mathrm{Br} J$ Ophthalmol 2000; 84: 1312-1317.

11. Bax DV, Mahalingam Y, Cain S, et al. Cell adhesion to fibrillin-1: identification of an Arg-Gly-Asp-dependent synergy region and a heparin-binding site that regulates focal adhesion formation. $J$ Cell Sci 2007; 120: 1383-1392.

12. Neptune ER, Frischmeyer PA, Arking DE, et al. Dysregulation of TGF $\beta$ activation contributes to pathogenesis in Marfan syndrome. Nat Genet 2003; 33: 407-411.

13. Warren R, Gartstein V, Kligman AM, et al. Age, sunlight, and facial skin: a histologic and quantitative study. J Am Acad Dermatol 1991; 25: 751-760.

14. Watson REB, Griffiths CEM, Craven NM, et al. Fibrillin-rich microfibrils are reduced in photoaged skin. Distribution at the dermal-epidermal junction. J Invest Dermatol 1999; 112: 782-787.

15. Kadoya K, Sasaki T, Kostka G, et al. Fibulin-5 deposition in human skin: decrease with ageing and ultraviolet $\mathrm{B}$ exposure and increase in solar elastosis. Br J Dermatol 2005; 153: 607-12.

16. Kunz J. Matrix metalloproteinases and atherogenesis in dependence of age. Gerontology 2007; 53: 63-73.

17. Meyer KC, Rosenthal NS, Soergel P, et al. Neutrophils and lowgrade inflammation in the seemingly normal aging human lung. Mech Ageing Dev 1998; 104: 169-181.

18. Chakraborti S, Mandal M, Das S, et al. Regulation of matrix metalloproteinases: an overview. Mol Cell Biochem 2003; 253: 269-285.

19. Clark IA, Swingler TE, Sampieri CL, et al. The regulation of matrix metalloproteinases and their inhibitors. Int J Biochem Cell Biol 2008; 40: 1362-1378.

20. Ashworth JL, Murphy G, Rock MJ, et al. Fibrillin degradation by matrix metalloproteinases: implications for connective tissue remodelling. Biochem J 1999; 340: 171-181.

21. Kielty CM. Catabolism of intact fibrillin microfibrils by neutrophil elastase, chymotrypsin and trypsin. FEBS Lett 1994; 351: 85-89.

22. Saarialho-Kere U, Kerkela E, Jeskanen L, et al. Accumulation of matrilysin (MMP-7) and macrophage metalloelastase (MMP-12) in actinic damage. J Invest Dermatol 1999; 113: 664-672.

23. Freeman LJ, Lomas A, Hodson N, et al. Accelerated publication fibulin-5 interacts with fibrillin-1 molecules and microfibrils. Biochem J 2005; 388: 1-5.

24. Baldock C, Koster AJ, Ziese U, et al. The supramolecular organization of fibrillin-rich microfibrils. J Cell Biol 2001; 152: 1045-1056.

25. Sherratt MJ, Bax DV, Chaudhry SS, et al. Substrate chemistry influences the morphology and biological function of adsorbed extracellular matrix assemblies. Biomaterials 2005; 26: 7192-7206.
26. Abramoff MD, Magelhaes PJ, Ram SJ. Image Processing with ImageJ. Biophoton Int 2004; 11: 36-42.

27. Horcas I, Fernandez R, Gomez-Rodriguez JM, et al. WSXM: a software for scanning probe microscopy and a tool for nanotechnology. Rev Sci Instrum 2007; 78: 013705.

28. Du H, Fuh RCA, Li JZ, et al. PhotochemCAD: a computer-aided design and research tool in photochemistry. Photochem Photobiol 1998; 68: $141-142$.

29. Kielty CM, Sherratt MJ, Marson A, et al. Fibrillin microfibrils. Adv Protein Chem 2005; 70: 405-436.

30. Sherratt MJ, Holmes DF, Shuttleworth CA, et al. Scanning transmission electron microscopy mass analysis of fibrillin-containing microfibrils from foetal elastic tissues. Int J Biochem Cell Biol 1997; 29: 1063-1070.

31. Young AR. Chromophores in human skin. Phys Med Biol 1997; 42: 789-802.

32. Downing AK, Knott V, Werner JM, et al. Solution structure of a pair of calcium-binding epidermal growth factor-like domains: implications for the Marfan syndrome and other genetic disorders. Cell 1996; 85: 597-605.

33. Yuan X, Downing AK, Knott V, et al. Solution structure of the transforming growth factor beta-binding protein-like module, a domain associated with matrix fibrils. EMBO J 1997; 16: 6659-6666.

34. Kerwin BA, Remmele RL. Protect from light: photodegradation and protein biologics. J Pharm Sci 2007; 96: 1468-1479.

35. Bergkvist M, Carlsson J, Oscarsson S. Surface-dependent conformations of human plasma fibronectin adsorbed to silica, mica, and hydrophobic surfaces, studied with use of atomic force microscopy. J Biomed Mater Res A 2003; 64: 349-356.

36. Hoffmann K, Kaspar K, Altmeyer P, et al. UV transmission measurements of small skin specimens with special quartz cuvettes. Dermatology 2000; 201: 307-311.

37. Diffey BL. Ultraviolet radiation and human health. Clin Dermatol 1998; 16: 83-89.

38. Christner PJ, Gentiletti J, Peters J, et al. Collagen dysregulation in the dermis of the $\mathrm{Sagg}^{+}$mouse: a loose skin model. J Invest Dermatol 2006; 126: 595-602.

39. Jennissen HP. Ubiquitin and the enigma of intracellular protein degradation. Eur J Biochem 1995; 231: 1-30.

40. Wlaschek M, Tantcheva-Poor I, Naderi L, et al. Solar UV irradiation and dermal photoaging. J Photochem Photobiol B Chem 2001; 63: $41-51$.

41. Shapiro SD, Endicott SK, Province MA, et al. Marked longevity of human lung parenchymal elastic fibers deduced from prevalence of D-aspartate and nuclear weapons-related radiocarbon. J Clin Invest 1991; 87: 1828-1834.

42. Ritz-Timme S, Laumeier I, Collins MJ. Aspartic acid racemization: evidence for marked longevity of elastin in human skin. $\mathrm{Br}$ J Dermatol 2003; 149: 951-959.

43. Wess J, Purslow PP, Sherratt MJ, et al. Calcium determines the supramolecular organization of fibrillin-rich microfibrils. $J$ Cell Biol 1998; 141: 829-837.

44. Cooper DR, Davidson RJ. Effect of ultraviolet irradiation on soluble collagen. Biochem J 1965; 97: 139-147.

45. Menter JM, Cornelison LM, Cannick L, et al. Effect of UV on the susceptibility of acid-soluble Skh-1 hairless mouse collagen to collagenase. Photodermatol Photoimmunol Photomed 2003; 19: 28-34.

46. Menter JM, Patta AM, Sayre RM, et al. Effect of UV irradiation on type I collagen fibril formation in neutral collagen solutions. Photodermatol Photoimmunol Photomed 2001; 17: 114-120.

47. Sionkowska A, Kaminska A. Thermal helix-coil transition in UVirradiated collagen from rat tail tendon. Int J Biol Macromol 1999; 24: $337-340$. 


\section{Selective protein degradation by ultraviolet radiation}

48. Garcia AJ, Vega MD, Boettiger D. Modulation of cell proliferation and differentiation through substrate-dependent changes in fibronectin conformation. Mol Biol Cell 1999; 10: 785-798.

49. Yamada KM. Cell-surface interactions with extracellular materials. Annu Rev Biochem 1983; 52: 761-799.

50. ${ }^{*}$ Birk DE, Bruckner P. Collagen suprastructures. In Collagen. Springer-Verlag: Berlin, 2005; 185-205.
51. *Iozzo RV. Matrix proteoglycans: from molecular design to cellular function. Аnnu Rev Biochem 1998; 67: 609-652.

*These references are cited only in the Supporting information online.

\section{SUPPORTING INFORMATION ON THE INTERNET}

The following supporting information may be found in the online version of this article:

Figure S1. STEM of UV irradiated cell culture and tissue derived fibrillin microfibrils.

Figure S2. Exposure to low doseUVR induces aggregation rather than fragmentation.

Figure S3. The influence of UVR on the electrophoretic mobility of type I collagen and fibronectin was invariant with substrate.

Figure S4. UVR on LDPE substrates induced aggregation but not fragmentation in susceptible proteins.

Figure S5. The influence of UVR on the area of adsorbed fibronectin monomers and dimers.

Figure S6. The relative susceptibility of ECM proteins to oxidation.

Table S1. UV chromophore content of dermal collagens.

Table S2. UV chromophore content of dermal proteoglycans and glycoproteins.

Table S3. UV chromophore content of dermal elastic fibre components. 